\title{
Le patrimoine méconnu des bois sacrés de la ville de Koudougou (Burkina Faso) : de la reconnaissance à la sauvegarde
}

\section{The unknown heritage of sacred woods in the town of Koudougou (Burkina Faso): Moving toward recognition and protection \\ El patrimonio desconocido de los bosques sagrados de la ciudad de Koudougou (Burkina Faso): Del reconocimiento a la salvaguardia}

\section{Lassane Yameogo}

\section{Volume 59, numéro 166, avril 2015}

Version originale soumise en février 2015. Version révisée reçue en septembre 2015.

\section{URI : https://id.erudit.org/iderudit/1034349ar}

DOI : https://doi.org/10.7202/1034349ar

Aller au sommaire du numéro

Éditeur(s)

Département de géographie de l’Université Laval

ISSN

0007-9766 (imprimé)

1708-8968 (numérique)

Découvrir la revue

Citer cet article

Yameogo, L. (2015). Le patrimoine méconnu des bois sacrés de la ville de

Koudougou (Burkina Faso) : de la reconnaissance à la sauvegarde. Cahiers de géographie du Québec, 59(166), 71-90. https://doi.org/10.7202/1034349ar

\section{Résumé de l'article}

Pour des raisons diverses, les sociétés rurales africaines ont organisé l'espace en privilégiant des lieux sacrés dotés d'interdits. Ces bois sacrés sont rattrapés par les dynamiques urbaines qui leur disputent l'espace. Le patrimoine que constituent ces jardins sacrés demeure inconnu du fait qu'il ne répond pas aux critères occidentaux du patrimoine culturel. Pour comprendre l'évolution des jardins sacrés et dans la perspective de leur valorisation, des entretiens auprès des responsables coutumiers et des services techniques complétés par une analyse spatiale ont été organisés à Koudougou. Il en ressort que ces jardins séculaires possèdent une grande biodiversité, mais sont fortement menacés par la pression foncière. Leur sauvegarde qui aurait pu provenir des structures modernes semble compromise en raison des mythes qui les entourent. Cependant, les acteurs reconnaissent que cet autre patrimoine culturel représente des lieux de mémoire dont la conservation et la valorisation contribueraient à l'entretien d'un cadre de vie sain. 


\title{
Le patrimoine méconnu des bois sacrés de la ville de Koudougou (Burkina Faso): de la reconnaissance à la sauvegarde
}

\author{
The unknown heritage of sacred woods \\ in the town of Koudougou (Burkina Faso): \\ Moving toward recognition and protection \\ El patrimonio desconocido de los bosques \\ sagrados de la ciudad de Koudougou (Burkina \\ Faso): Del reconocimiento a la salvaguardia
}

\author{
Lassane YAMEOGO \\ Département de géographie \\ Université de Ouagadougou \\ Yameogolass@gmail.com
}

\section{Résumé}

Pour des raisons diverses, les sociétés rurales africaines ont organisé l'espace en privilégiant des lieux sacrés dotés d'interdits. Ces bois sacrés sont rattrapés par les dynamiques urbaines qui leur disputent l'espace. Le patrimoine que constituent ces jardins sacrés demeure inconnu du fait qu'il ne répond pas aux critères occidentaux du patrimoine culturel. Pour comprendre l'évolution des jardins sacrés et dans la perspective de leur valorisation, des entretiens auprès des responsables coutumiers et des services techniques complétés par une analyse spatiale ont été organisés à Koudougou. Il en ressort que ces jardins séculaires possèdent une grande biodiversité, mais sont fortement menacés par la pression foncière. Leur sauvegarde qui aurait pu provenir des structures modernes semble compromise en raison des mythes qui les entourent. Cependant, les acteurs reconnaissent que cet autre patrimoine culturel représente des lieux de mémoire dont la conservation et la valorisation contribueraient à l'entretien d'un cadre de vie sain.

\section{Mots-clés}

Burkina Faso, Koudougou, bois sacrés, valorisation, patrimoine.

\begin{abstract}
For a variety of reasons, rural African societies have organized their space to include certain sacred areas subject to particular prohibitions. An example can be found in sacred gardens or woods, today confronted by an urban dynamic laying claim to those very same spaces. The heritage value of these sacred gardens remains unknown because they do not correspond to Western cultural heritage criteria. To understand the evolution of such sacred spaces and potentially improve their status, survey interviews were carried out with traditional community leaders and certain technical services in Koudougou, together with a spatial analysis. The results show that these ancient gardens have high biodiversity but are strongly threatened by pressure for land. Their protection, which might have been assured by modern community structures, seems to be in jeopardy because of the myths surrounding them. However, the people involved recognize that these different forms of cultural heritage represent places of memory whose preservation and increased value could be a significant factor in maintaining a healthy living environment.
\end{abstract}

\section{Keywords}

Burkina Faso, Koudougou, sacred woods, increased value, heritage. 


\section{Resumen}

Por diversas razones, las sociedades rurales africanas han organizado el espacio privilegiando lugares sagrados objeto de tabúes. Esos bosques sagrados están siendo recuperados por dinamismos urbanos que les disputan el espacio. Esos jardines sagrados no responden a criterios culturales occidentales, por lo tanto, son totalmente ignorados. Para comprender la evolución de los jardines sagrados valorizándolos, se realizaron, en Koudougou, entrevistas con responsables consuetudinarios y con los de servicios técnicos, las que se completaron con un análisis espacial. Resulta que esos jardines seculares poseen grande biodiversidad, siendo, sin embargo, fuertemente amenazados por la presión inmobiliaria. Su protección, que podría provenir de estructuras modernas, estaría a riesgo por los mitos que les circundan. Pero, los actores reconocen a este patrimonio cultural un valor de espacios de reminiscencia, cuya conservación y valorización contribuirían al mantenimiento de un medio de vida sana.

\section{Palabras claves}

Burkina Faso, Koudougou, bosques sagrados, valorización, patrimonio.

\section{Introduction}

La préservation des ressources naturelles a toujours constitué une préoccupation majeure dans les sociétés africaines. Elle s'est manifestée sous diverses formes, dont les plus visibles et encore d'actualité sont les forêts sacrées. Ces espaces sacrés, jadis situés dans des milieux ruraux, sont aujourd'hui phagocytés par les agglomérations et posent ainsi des problèmes de gestion dans des milieux où l'urbanisation rapide est l'une des causes principales de dégradation de l'environnement. Aussi, ce phénomène est-il une entrave à la conservation des espaces verts et des lieux sacrés. Ces enclaves sont traditionnellement des lieux de culte et d'initiation. En cela, elles sont l'objet d'une totale protection par les populations des villages, nonobstant les menaces que laissent présager les pressions diverses, humaines et animales.

L'urbanisation de la commune de Koudougou est source de mutations spatiales, socioéconomiques et culturelles, ainsi que de dégradation du couvert végétal, affectant les espaces boisés qui s'y trouvent. Ces derniers, qualifiés de forêts sacrées ou bois sacrés ${ }^{1}$, ont longtemps bénéficié de la protection des populations autochtones. Cette protection selon les us et coutumes a permis d'éviter leur disparition. Aujourd'hui, cependant, les forêts sacrées font face à une pression foncière combinée à l'évolution des pratiques culturelles. Cette situation pose l'épineuse question de leur gestion. Au regard des nombreux problèmes et enjeux auxquels sont confrontées les forêts sacrées de Koudougou, on peut s'interroger sur des modes de gestion probables de ces espaces dans un contexte de mutations socioéconomiques et culturelles. Comment peut-on gérer durablement une forêt sacrée dans un contexte urbain et semi-urbain? Quels acteurs faut-il alors impliquer? Existe-t-il de réelles possibilités d'aménagement de ces espaces pour en faire des lieux patrimoniaux? Le rapport spirituel à la nature peut-il

1 Dans l'analyse qui suit, les termes «bois sacré» et «forêt sacrée» désignent les espaces boisés destinés à l'initiation ou à la conservation des fétiches, ou encore des endroits pour rendre des cultes aux ancêtres. Dans cette acception, la superficie du site n'a pas une importance significative; c'est plutôt la fonction symbolique qui est significative à notre sens. 
induire des décisions de gestion territoriale de la nature? Trois objectifs guident cet article: inventorier les formations sacrées et les logiques de leur conservation par les populations et la municipalité; déterminer les menaces qui pèsent sur la sauvegarde des forêts sacrées; et analyser les possibilités d'aménagement des forêts sacrées dans une perspective de patrimonialisation.

\section{Site d'étude et démarche méthodologique}

\section{Les sites de la recherche}

La commune de Koudougou s'étend sur $272 \mathrm{~km}^{2}$ et totalise 10 secteurs avec des caractéristiques allant de l'urbain au rural (ONU-Habitat, 2005), les espaces périurbains étant plus à dominance rurale (figure 1). La commune possède des espaces gérés traditionnellement, parmi lesquels les bois sacrés. Ces entités subissent de plus en plus le poids de la ville.

\section{La démarche méthodologique}

Dans la perspective de réunir et traiter les données permettant d'atteindre les objectifs fixés, la méthodologie, tout en privilégiant une approche qualitative et quantitative, a consisté d'abord en la recherche et en l'exploitation de la documentation relative à la dynamique urbaine et au mode de gestion traditionnelle de l'environnement. Elle a ensuite été complétée par une série d’observations in situ et par l'administration (i) de questionnaires à une centaine de ménages ${ }^{2}$, essentiellement des riverains des bois sacrés, (ii) d'entretiens semi-directifs avec des responsables coutumiers, des responsables administratifs et du personnel des services de l'environnement. Cent vingt ménages ont ainsi été sélectionnés par choix aléatoire en fonction du nombre de bois sacrés par quartier et des parcelles riveraines. Autour de chaque îlot de végétation, il y a en moyenne 32 parcelles d'habitation censées accueillir au moins deux ménages. Dans les quartiers disposant de deux bois sacrés (Zinguédéguin et Dapoya), 40 ménages par quartier ont été interviewés. À Sogpelcé, quartier disposant d'un seul bois sacré, 20 ménages ont été rencontrés et, enfin, dans chacun des quartiers ne disposant pas de bois sacré (Bourkina et Issouka), 10 ménages ont accordé des entretiens. Ces derniers secteurs ont été considérés comme des sites témoins permettant d'apprécier les disparités spatiales et les différences de perception pouvant être liées à la présence ou à l'absence d'un bois sacré. Les guides d'entretien ont été adressés aux responsables des collectivités territoriales, au responsable du service de l'environnement et aux notables coutumiers ; huit de ces personnes ont été interviewées.

Ces informations ont été complétées par une collecte de données à référence spatiale des sites de bois sacrés dans le centre urbain et dans les milieux ruraux périphériques. Le traitement des données spatiales et des enquêtes au moyen d'Arcview 3.2 et de Sphinx ont permis de faire les analyses qui suivent.

2 Un ménage au Burkina Faso est en moyenne composé de sept personnes selon l'enquête démographique et santé de 2013 . 
Figure 1 Localisation des quartiers et villages périphériques de la commune de Koudougou

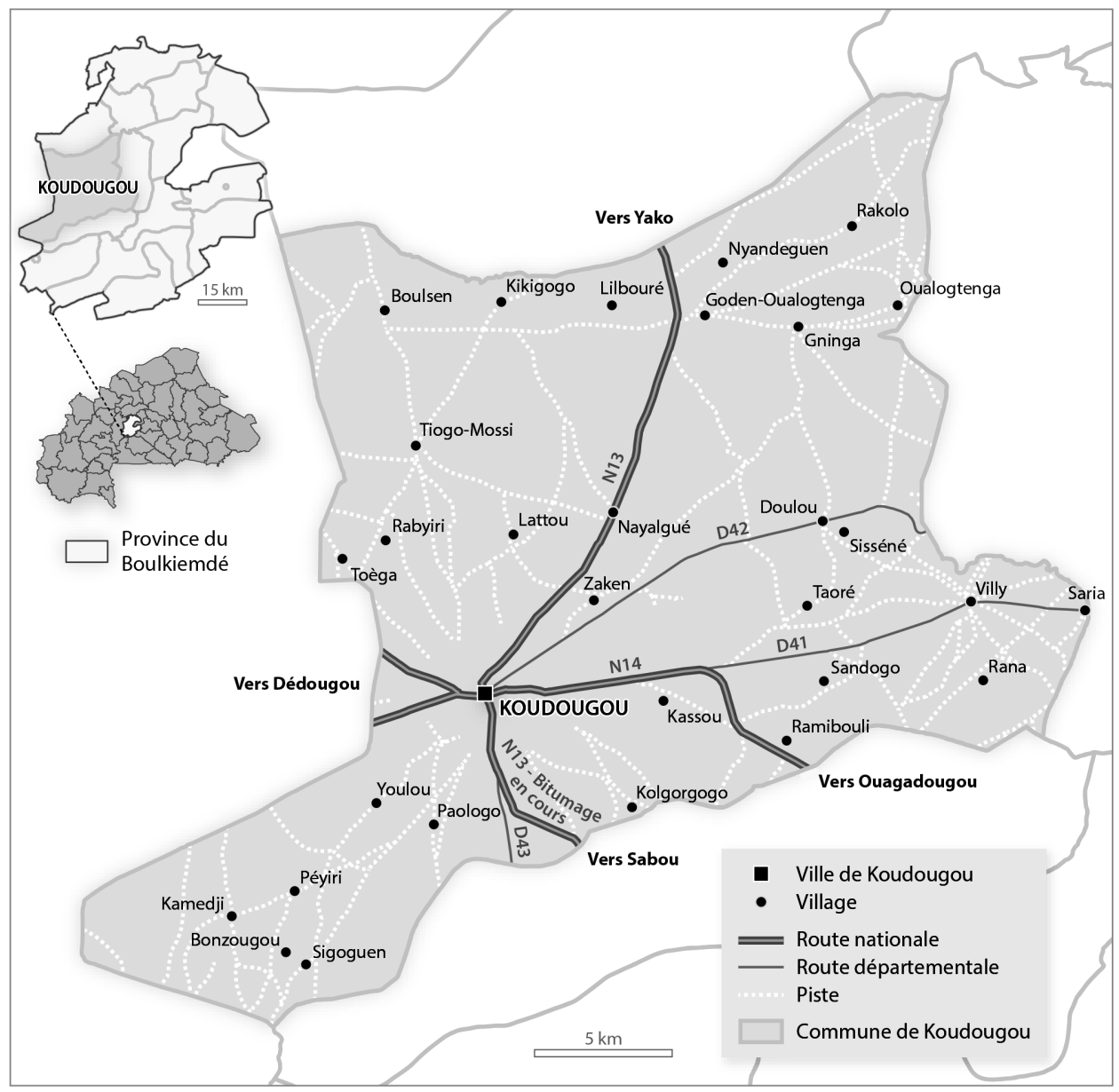

Source: IGB/BNDT 2002

Réalisation: Lassane Yameogo, 2015

Adapté par le Département de géographie de l'Université Laval

\section{Le sacré dans l'appropriation symbolique et le contrôle territorial}

Les motivations pour la protection des ressources naturelles s'appuient sur des questionnements divers, tendant à déterminer les valeurs à accorder à la nature et le niveau que l'action humaine peut atteindre dans sa relation avec la nature. Elles traduisent la conception que les sociétés ont de la conservation. Dans l'action de conserver figure une notion essentielle, qui est celle de maintenir en l'état un phénomène ou une ressource. L'application de la conservation à la nature implique donc une gestion dont les finalités sont de préserver une des espèces faunique ou floristique, voire un paysage considéré comme menacé. Depuis les premières réserves de Yellowstone aux États-Unis, une mobilisation constante accompagne les forêts classées et les aires protégées. À l'inverse, les espaces sacrés ne font l'objet d'une attention particulière que depuis l'adoption de la convention sur la diversité biologique, en 1992 (Ibo, 2005). Jadis vilipendées par les adeptes des religions monothéistes du fait des pratiques 
magicoreligieuses qui s’y déroulent, les forêts sacrées sont de plus en plus considérées comme des espaces de biodiversité à protéger. Avec le temps, on se rend compte que cette forme de protection par la sacralisation des sites, produit de bons résultats au regard de l'implication des acteurs (Ibo, 2005; Juhé-Beaulaton, 2006). La protection est essentiellement liée à la perception que les différents groupes sociaux ont de leur milieu. Dans les sociétés rurales, notamment en Afrique, certaines règles ou certains interdits sont établis par les populations locales en vue d'une conservation durable des ressources de la nature à leur profit. Spécifiquement chez les Mossé, certaines pratiques dans la société révèlent à quel point la dimension religieuse est créatrice de paysages naturels.

\section{Une géographie religieuse de l'espace, protectrice de l'environnement}

La géographie religieuse se focalise sur le rôle du religieux dans la formation des perceptions humaines du monde et de la place qu'y tient l'humanité (Deffontaines, 1948; Racine et Walther, 2003). Dans la société moaga ${ }^{3}$, l'arbre n’a pas seulement une fonction utilitaire, car tout un ensemble de représentations sociales et spirituelles lui sont liées. L'arbre y apparaît comme l'image vivante de l'homme (Kaboré, 1987). Il n'est pas seulement un élément végétal détaché de la réalité humaine, il lui est consubstantiel. Certaines espèces végétales seraient ainsi douées d'un côté religieux et sacré fortement pris en compte dans les différents usages sociaux. Chez les Mossé du Kouritenga, confier un enfant au néré (Parkia biglobosa) confère à ce dernier les caractéristiques d'une entité qui possède des vertus mystérieuses bénéfiques ou abrite des génies protecteurs. En outre, les liens entre l'homme et la végétation, voire l'environnement dans son ensemble, se manifestent également à travers les morts. Kaboré (1987) et Barreteau et al. (1991) confirment à cet égard que les morts dont on n’a pas pu rapporter la dépouille au village en raison d'un éloignement excessif, d'une disparition par noyade ou au cours de combats, sont, dans un grand nombre d'ethnies, remplacés par un fragment ligneux symbolique. L’arbre est donc considéré comme un véritable substitut du corps défunt. Aussi, sera-t-il l'objet de funérailles avant d'être inhumé. Ailleurs, chez les Sonraï, le jujubier (Ziziphus mauritiana) est employé dans les cérémonies funèbres. Les feuilles sont utilisées pour la toilette des morts, tandis que les branches servent de bois-tombaux. Quant aux fruits, ils seraient sucés par les enfants au paradis (Ganaba et al., 1998), tel que cité par Hien (2007)). De ce fait, le jujubier est une plante hantée par les esprits, les bons génies et les djinns ${ }^{4}$ des enfants (c'est pourquoi on l'utilise pour délivrer les femmes en difficulté d'accouchement).

À travers cette géographie religieuse, la végétation est perçue par plusieurs sociétés rurales comme un élément de la nature doté de pouvoirs surnaturels et avec lequel il faut composer. À Koudougou, elle joue un rôle allant du plus utilitaire au religieux. L'arbre y est conçu comme un «être» utile (ombre, bois, médicaments, etc.), mais surtout mystique et doté de pouvoirs magiques. Dès lors, l'espace qui abrite ces pouvoirs sera également investi de facultés qui en font un lieu sacré. Une telle perception de l'espace est moins intéressante par ses formes et ses structures que par sa symbolique. C'est en effet la reconnaissance de cet espace qui lui confère une dimension sacrée et qui, de ce fait, demeure au centre des considérations propres aux religions traditionnelles.

3 Les Mossé constituent le groupe ethnique majoritaire au Burkina Faso (Moaga en est le singulier). Ils occupent la partie centrale du pays.

4 Esprit bienfaisant, génie ou démon, dans les croyances musulmanes. 
De nombreux arbres sont totalement interdits de coupe; même quand ils sont morts et secs, leur utilisation nécessite des précautions (figures 2 et 3). Seuls les «coutumiers» ont le droit, après certaines offrandes, d'en ramasser les branches sèches tombées d'ellesmêmes. Ils les gardent en tas et ne les utilisent que pour les feux rituels. Selon la tradition, l'utilisation de ce bois par une femme non ménopausée entraîne la perte de la fertilité.

Les arbres rares des savanes sont souvent considérés comme lieux de refuge des génies dont il serait dangereux de s'attirer les foudres (CIRAD, 2001). C'est ainsi que des espèces telles que kankanga (Ficus gnaphalocarpa), kankalga (Daniela oliveri), pusga (Tamarindus indica), siiga (Anogeissus leiocarpa) sont reconnues par l'ensemble des responsables coutumiers interrogés comme étant des espèces sacrées. La plupart de ces arbres abriteraient des esprits qui sont souvent vénérés. Chaque arbre possède ses génies, ses remèdes et ses sorts (Bonet, 1984, cité par Kaboré, 1994). Cela justifie les sacrifices offerts à ces végétaux qui, en retour, accordent aux donateurs, par l'entremise des entités surnaturelles qui les habitent, ce dont ils ont besoin, protection, santé et progéniture (figures 2 et 3 ). Ainsi, ce lien qui existe entre les hommes et les divinités apparaît comme un rapport de réciprocité, de don contre don ou de marché avec marchandage. Les interdits entourant certaines espèces végétales ont ainsi permis d'assurer la protection des îlots de forêt environnants. Pour Duchesne (2002), en raison des vertus qu'on leur prête, des lieux de refuge et de reproduction pour la faune et pour la flore sont préservés.

\section{Figure 2 Kaya sénégalensis sacré, dans le secteur 3}

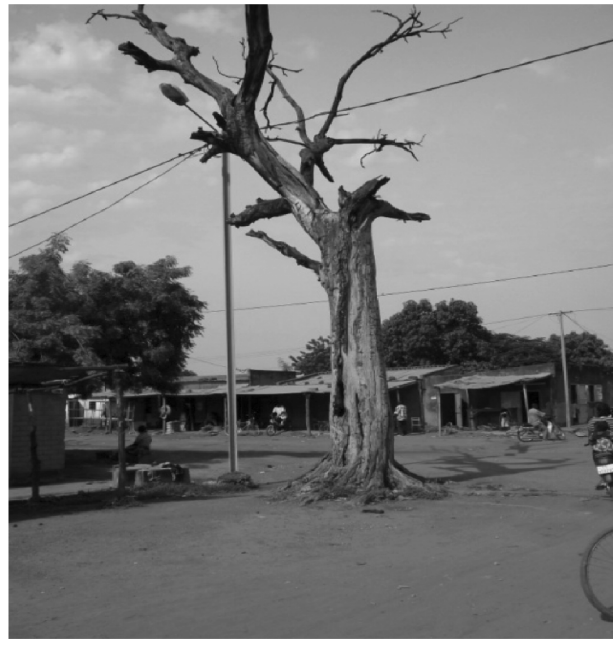

Photo: Bayala E., septembre 2010

\section{Figure 3 Arbre (Daniela oliveri) sacrificiel, dans le secteur 3}

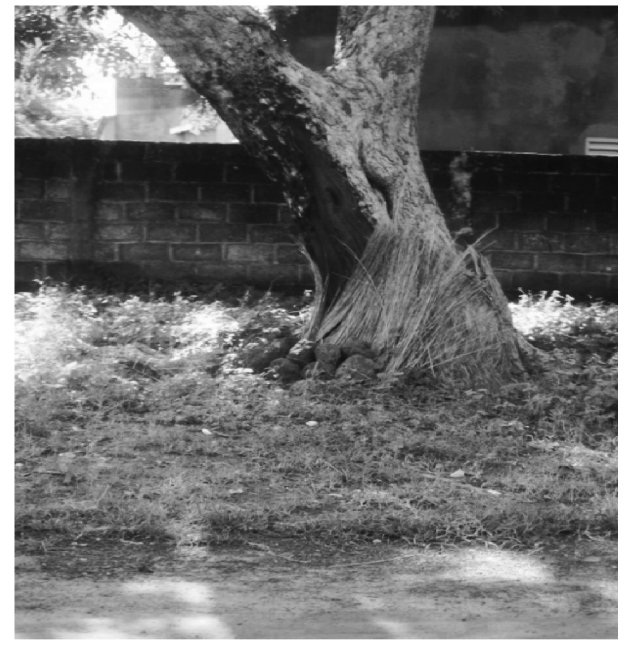

Photo: Bayala E., septembre 2010

\section{Le rôle protecteur de l'arbre}

Dans la croyance traditionnelle, nonobstant leur rôle utilitaire, les arbres - et en particulier ceux investis par des entités invisibles - restent des moyens privilégiés par lesquels l'homme peut entrer en contact avec les ancêtres, les génies, les divinités. Ils 
sont les refuges des «dieux». En effet, ces entités surnaturelles y vivent et ont aussi besoin de l'assistance humaine, sans laquelle elles pourraient se mettre en colère. Cela se traduit de nos jours par la présence d'enclaves boisées traditionnellement protégées au sein des villages et des villes.

Les travaux de terrain ont révélé la présence de cinq forêts sacrées à Koudougou. Celles-ci sont réparties dans trois quartiers, à savoir deux forêts sacrées à Dapoya (Sebgtanga et Bougatanga), deux à Zinguédéguin (Koongo et Tanga) et une à Sogpelcé (figure 4). Selon 13,8\% des personnes interviewées, elles constituent des sanctuaires, tandis que les autres participants à l'enquête les considèrent comme de simples espaces boisés. Pour les premiers, l'aspect magicoreligieux de l'environnement a motivé la conservation de tels espaces.

L'existence de ces massifs forestiers témoigne de l'intérêt que les sociétés traditionnelles portaient à la protection de l'environnement. Elle laisse entrevoir chez celles-ci un souci de gestion religieuse des ressources naturelles qui, de nos jours, se confond avec une gestion durable apparue avec les crises écologiques. En effet, la manière de gérer les ressources environnementales, de les utiliser et de les protéger, par la société traditionnelle, découle de la perception qu'à celle-ci de l'environnement. De ce fait, selon le ministère de l'Environnement et du cadre de vie (MECV, 2006), l'arbre est considéré comme un être vivant au service de l'homme et occupe une place importante dans l'univers existentiel des sociétés rurales. L’environnement y serait perçu comme un don divin confié aux humains pour le gérer à leur profit.

En outre, la position périphérique des massifs de bois sacrés par rapport aux villages peut être lue comme une fonction de défense: les bois sacrés servaient de site de refuge en cas d'attaque du village et pouvaient également limiter l'accès des cavaleries (Pélissier, 1980).

\section{Le totémisme comme forme de conservation}

La conception africaine de la vie établit une relation de continuité entre l'homme et son environnement naturel, entre le monde matériel visible et le monde spirituel invisible. Pour Agnissan (2010), «le caractère sacré de ce continuum Homme-Nature s'exprime à travers les totémismes, les rites agraires, les pratiques liturgiques dont les supports écologiques demeurent souvent les bois ou les forêts sacrées». La logique de protection de la nature par des interdits ou la sacralisation de certaines espèces animales et végétales ou de certains paysages tire ses origines de cette relation homme-nature.

Pour les autochtones de Bourkina, par exemple, le baobab (Adansonia digitata) est un totem. Sa germination dans le domaine d'une personne originaire du quartier nécessite que cette personne le protège. Ce totem serait lié au premier ancêtre qui aurait bénéficié des bonnes grâces d'un baobab habité par des esprits, à côté duquel l'ancêtre se serait installé. Ce baobab (l'un des rares dans le rayon) existe encore aujourd'hui et fait l'objet d'un culte (figures 5 et 6). Le sacré n'est pas uniquement lié aux ressources végétales, d'autres éléments de la nature tels que les cours d'eau ou les mares font l'objet de protection et de sacralisation. 
Figure 4 Répartition spatiale des forêts sacrées à Koudougou

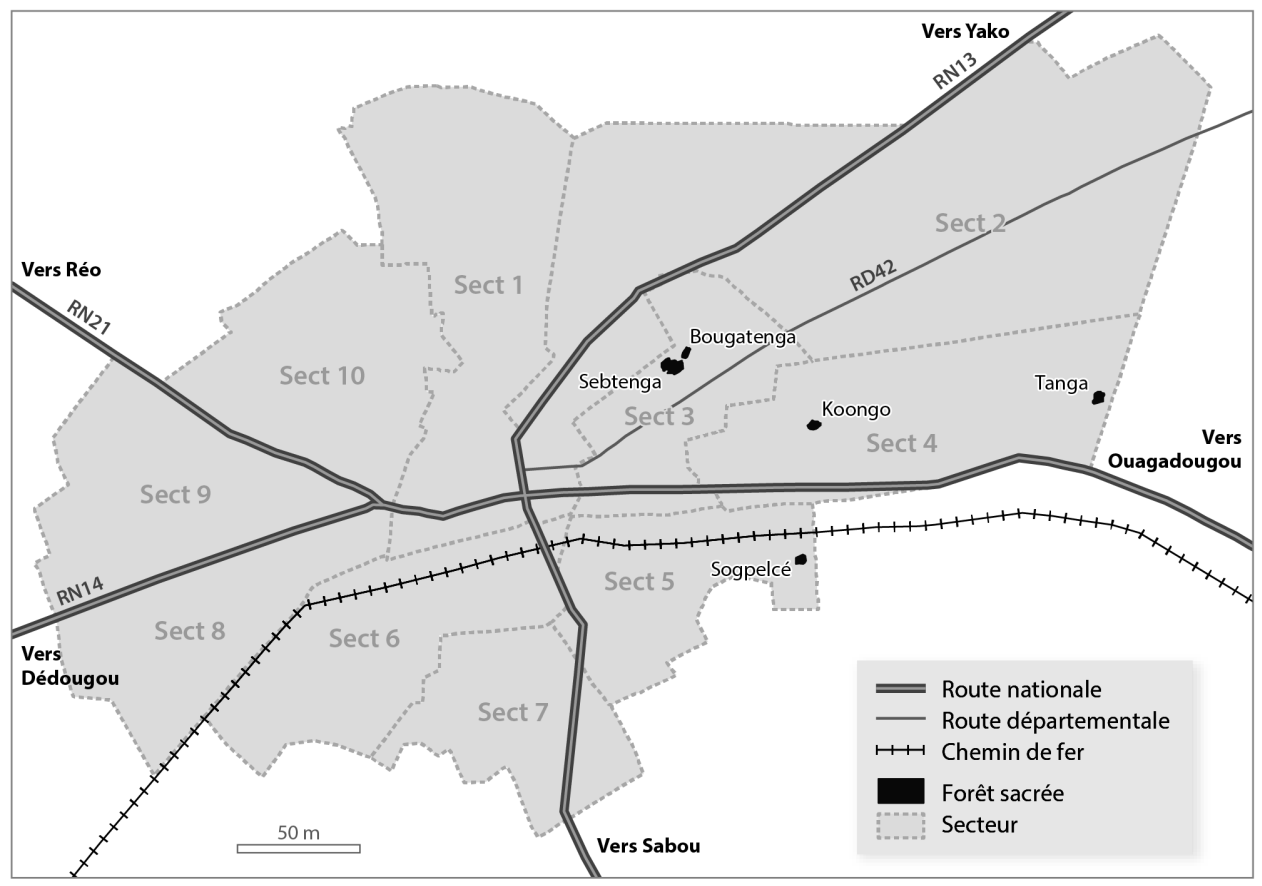

Source: IGB/Mairie de Koudougou et enquête terrain Réalisation: Lassane Yameogo, 2015

Adapté par le Département de géographie de I'Université Laval

Figure 5 Baobab (Adansonia digitata) sacré

Photo: Bayala E., septembre 2010

\section{du quartier Bourkina,
dans le secteur 9 \\ du quartier Bourkina
dans le secteur 9}

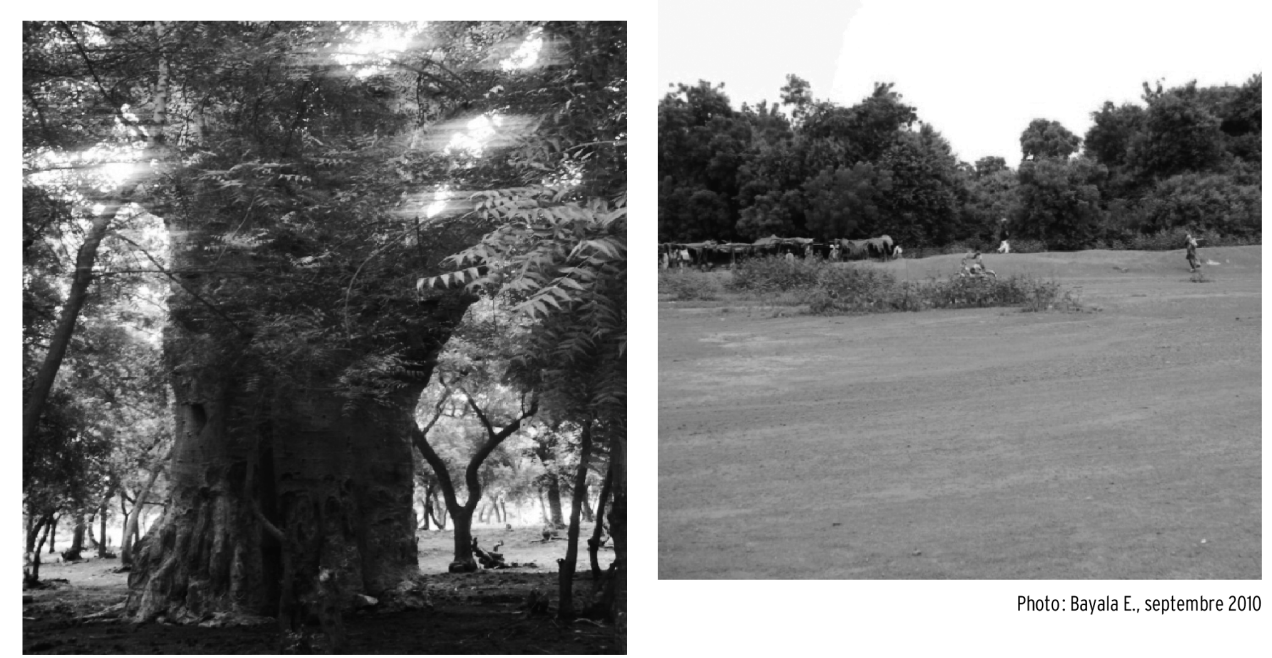

Figure 6 Forêt sacrée « Koongo »

Photo: Bayala E., septembre 2010 


\section{Le sacré de l'eau}

L'eau, source de vie mais aussi de mort, a toujours comporté cette idée d'ambivalence dans la perception que les sociétés se font d'elle à travers le monde. Elle a une action bienfaitrice (boisson, purification), mais peut être un élément de punition (le déluge, selon la bible). Dans les sociétés africaines, l'eau a toujours revêtu une symbolique importante. À l'image des religions monothéistes ${ }^{5}$, la symbolique de l'eau dans les religions traditionnelles se manifeste par le fait que de nombreuses divinités s'y cachent.

C'est ainsi qu'à Koudougou, certaines divinités ont pour lieu «d'habitation » des cours d'eau. Leur présence en ces lieux requiert des cérémonies et rites traditionnels. Des points d'eau comme le marigot de Bourkina, le marigot sacré Rita samba (figure 7) et le puits Bul-kiongo passent ainsi pour des lieux mystiques. En effet, les responsables coutumiers de Bourkina reconnaissent le marigot dudit quartier comme un de leurs lieux de culte. Ce site abriterait des génies (mâle et femelle).

En ce qui concerne le marigot Rita samba, il est la propriété culturelle des autochtones de Sogpelcé. Ce marigot aurait la particularité de noyer toute personne ayant commis une aberration ou qui est animée d'un esprit maléfique (sorcier, tueur, etc.) et qui y entrerait ou s'en approcherait de trop près.

\section{Figure 7 Marigot Rita samba, dans le secteur 5 de Koudougou}

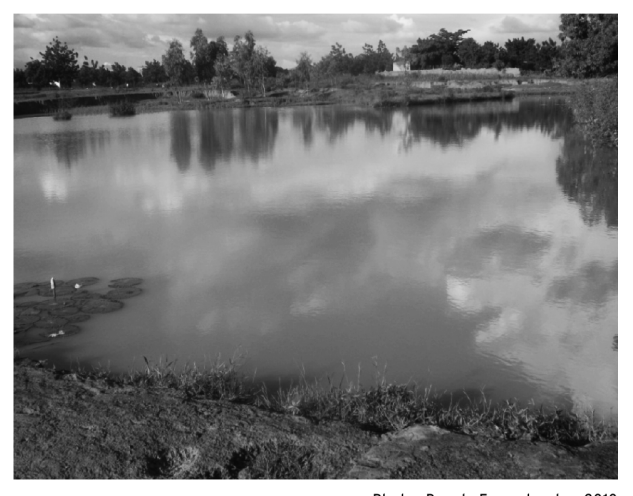

Le Bul-kiongo (puits intarissable), éponyme de la province du Boulkiemdé, quant à lui, est le sanctuaire de la population d'Issouka. Selon les coutumiers, il serait le premier puits de Koudougou. Intarissable et purificateur, il sert de point de contact entre les hommes et les ancêtres. De ce fait, des sacrifices y sont souvent faits pour implorer l'assistance des aïeux.

L'eau intervient dans tous les domaines d'activités. Aussi bien dans le monde visible (hommes, plantes, animaux) que dans le monde invisible (ancêtres, génies, esprits, dieux), elle est sollicitée pour ses qualités vitales. À ce sujet, Boukpessi (2010) soutient que les morts ont les mêmes besoins que les vivants: ils mangent, boivent, etc. Cette nécessité de l'eau s'illustre par son utilisation lors des sacrifices offerts aux divinités. Selon le rituel, elle peut être offerte sous sa forme naturelle, sous forme de dolo (bière locale à base de sorgho) ou sous forme de zoom-koom (sucrerie faite à base de farine de petit mil).

L'eau apparaît comme une ressource capitale, tant chez l'homme que chez les divinités. Son coté purificateur est largement utilisé dans les religions polythéistes. Son utilisation dans la société revêt deux formes: la forme utilitaire (alimentation, activités) et la religieuse (sacrifices, purification). Ce deuxième mode d'utilisation est ainsi une garantie de l'usage rationnel de la ressource précieuse. Le culte des marigots

5 Les musulmans l'utilisent dans la purification avant la prière, tandis que le baptême chrétien est réalisé avec de l'eau. 
et les interdits qui les entourent ont limité l'accès des habitants à ces sites, mais aussi aux espaces mitoyens. Ainsi, dans un rayon avoisinant $300 \mathrm{~m}$, une végétation assez luxuriante s'est développée autour du cours d'eau et des marigots. Au-delà du fait que cette zone est non aedificandi, la protection assurée par le caractère sacré a favorisé le développement d'îlots de végétation.

Le contrôle des ressources naturelles (eau, végétation) est, par le culte qui leur est voué, sous le contrôle du lignage fondateur. Cette alliance religieuse avec les ressources naturelles fonde donc l'appropriation et une assise politique au lignage fondateur. Le sacré qui entoure ces deux ressources a permis la conservation des espaces les plus boisés dans l'agglomération.

\section{Les bois sacrés: des espaces de biodiversité}

La pharmacopée traditionnelle est un des motifs valables de la conservation de la biodiversité floristique. Elle a permis à de nombreuses espèces d'échapper à la disparition, de contribuer à la préservation d'enclaves boisées. Le siiga (Anogeissus leiocarpa), par exemple, est devenu très rare dans la zone d'étude; cependant, il constitue l'essentiel de la formation végétale de la forêt sacrée de Sogpelcé. La société conserve ainsi jalousement ses reliques forestières par souci de garder des espèces végétales utiles et nécessaires pour sa survie. Cette protection doit sa rigueur aux interdits sociaux édictés à cet effet: interdiction de coupe, interdiction d'y aménager des habitations, interdiction de ramassage du bois mort (sauf les coutumiers pour le rituel), etc.

\section{L'organisation du territoire par le sacré et les défis de la gouvernance des bois sacrés}

L’analyse du concept de sacré dans la société moaga, ainsi que de ses manifestations, révèle que la sacralisation des lieux procède d'un processus qu'on pourrait qualifier de territorialisation, qui est la marque géographique de la construction sociale (Turco, 1988). La religion traditionnelle, maîtresse d'œuvre de cette construction sociale du sacré, permet d'abord de le penser, de l'organiser et enfin d'en assurer le contrôle.

\section{La territorialisation du sacré}

Les bois sacrés préservés dans les sociétés agraires, au regard des interdits dont ils font l'objet, ont réussi à s'imposer dans le paysage d'abord par une construction sociale. En effet, penser le monde à travers le sens, la symbolique des lieux ou des objets est une preuve de la volonté d'imposer un certain ordre à l'espace et à la société. Il y a donc une appropriation symbolique de l'espace dont la résultante est le fait de marquer une différence entre le sacré et le profane. Pour Racine et Walther (2003), «en tant qu'essence du religieux, le sacré indique des interdits et des attachements fondamentaux pour l'existence humaine». La société dans son ensemble reste marquée par le respect dû à ces espaces sacrés.

Loin de se limiter à penser l'espace sacré, les sociétés agraires impriment leur marque sur cet espace en procédant à sa délimitation, voire sa réification. Ainsi, les lieux colonisés par les dieux sont séparés des lieux dits profanes. Les lieux sacrés, 
délimités et structurés, abritent une végétation particulièrement dense par rapport au paysage environnant. D’autres sites aquatiques, tels que les marigots et cours d'eau, bénéficient également de cette réification de l'espace sacré. Dans les deux cas, tous les sites sont investis de la symbolique du sacré. La position géographique des lieux sacrés et leur symbolique ne sont pas le fruit du hasard; elles répondent à une certaine logique, proche de l'idéal divin. La ville de Koudougou, dans ses limites anciennes, est cernée par les lieux sacrés. La position géographique de ces lieux, autour des quartiers anciens ${ }^{6}$, montre le lien que les communautés entretiennent avec les bois sacrés. La lecture qui s'en dégage révèle une ville qui doit sa dynamique à la disposition périphérique des lieux sacrés censés assurer sa protection. La proximité des quartiers (Bourkina, Sogpelcé et Issouka) des sites sacrés comme le marigot de Bourkina, le marigot sacré Rita samba et le puits Bul-kiongo semble avoir présidé à l'agglomération de ces localités. S'inspirant d'exemples dans l'histoire des villes, Racine (1993) a montré de quelles manières le rapport au sacré ou au religieux a été à l'origine de certaines cités et au développement d'agglomérations urbaines. En rappel, d'autres auteurs à l'image de Pélissier (1980) évoquent cette position des massifs forestiers à la périphérie des villages comme une fonction de défense. Les bois sacrés à la périphérie des quartiers de Koudougou auraient ainsi pu servir de refuge pour les habitants en cas d'attaque des villages.

La troisième facette est le contrôle et l'influence que l'espace sacré exerce sur les territoires et les acteurs. Pour Racine et Walther (2003), "en même temps que les signes structurent l'espace du sacré et du symbolique, ils permettent le contrôle des hommes et contribuent à l'émergence de nouvelles spatialités où le politique et le religieux s'unissent». La société traditionnelle est organisée de façon à promouvoir le maintien de l'équilibre de l'environnement. Mais au-delà de l'environnement, c'est même l'équilibre de la société qui est maintenu à travers cette alliance entre politique et sacré. Les acteurs politiques et les tenants du religieux traditionnel s'associent dans la gestion du sacré, et donc dans la préservation des ressources naturelles. Les différentes instances de gestion assurent un contrôle de l'utilisation rationnelle des ressources naturelles par la sacralisation. Ces lieux sacrés sont sous la responsabilité des chefs coutumiers, qui ont la charge d'en assurer la protection et la survie. De ce fait, les chefs veillent au respect des traditions et lois qui régissent ces endroits en usant du pouvoir dont ils ont hérité. Leurs actions qui visent la conservation de ressources naturelles à caractère culturel permettent la sauvegarde des forêts sacrées. La gestion des bois sacrés à Koudougou fait appel à trois niveaux de gestion: le chef de canton, les chefs de quartiers et les tengsoba (chefs de terre). Le chef de canton, dont l'emprise politique s'étend sur tout le canton, est la première autorité qui préside à la gestion de l'environnement, notamment les ressources naturelles sacrées. Il joue un rôle d'arbitre et règle les conflits qui surgissent en matière d'utilisation des ressources de la nature. À la suite du chef de canton, les chefs de quartiers sont les garants de la protection des lieux sacrés du ressort de leur territoire. Ils sont assistés par les tengsoba. Ces derniers sont les premiers occupants des lieux et traitent des questions foncières et des sacrifices rituels pour implorer les dieux ou leur intercession. Ils sont par ailleurs détenteurs de toute l'information concernant les différentes propriétés, les lieux sacrés, les règles et interdits qui les régissent, les divinités, etc. Ils sont les premiers recours en cas de conflits fonciers, de rites liés aux bois sacrés, etc.

6 Ce sont les quartiers des lignages fondateurs de la ville. 
Mais avec l'accroissement de la population, de l'urbanisation et, surtout, l'implantation des religions monothéistes, ils font face à de nouveaux défis qui imposent de nouveaux mécanismes de gouvernance et de nouvelles fonctionnalités. Comment ces espaces sont-ils gérés dans ce nouveau contexte?

\section{Les bois sacrés en péril et la réappropriation par de nouveaux acteurs}

L’urbanisation galopante des localités du Burkina Faso et l'émergence de nouveaux rapports aux croyances (multiplication des églises et mosquées, apparition de sectes), la retraduction du divin en milieu urbain amènent à repenser le rapport à l'espace sacré et aux ressources naturelles qu'il abrite. De ce fait, elles induisent de profonds changements socioéconomiques et des bouleversements dans l'équilibre des écosystèmes. Il s'agit des mutations qui se sont produites au sein de la société et qui seraient des conséquences d'une croissance urbaine de la commune. Ces mutations ont trait à la démographie urbaine, aux mœurs et aux coutumes.

\section{Les facteurs du changement de paradigme dans la gestion des bois sacrés}

\section{L'augmentation de la population}

La population de Koudougou a connu une évolution spectaculaire, passant de 15920 habitants en 1936 à 138209 en 2006 (Konsegré, 2007). Une telle croissance démographique s'est accompagnée d'une extension du territoire communal, réduisant ainsi les espaces verts au profit des lotissements et équipements urbains. L'accroissement urbain s'est matérialisé par une concentration de populations de diverses cultures entraînant des différences d'appréciation de la valeur de la nature et de la conservation. En effet, les forêts sacrées sont soumises à une pression anthropique qui rend leur gestion problématique. Le brassage culturel entre population locale et allochtone perturbe l'équilibre instauré par les autochtones, en rapport avec les forêts sacrées. La différence de cultures, de mœurs et de perceptions joue négativement sur le respect qui est voué à ces espaces.

La forte concentration de population, la diversité ethnique et religieuse constituent donc un handicap majeur pour la préservation d'espaces verts par les méthodes traditionnelles. Les forêts sacrées sont par conséquent en proie à de sérieuses contraintes de gestion. À ce propos, Juhé-Beaulaton (2009) soutient que ces lieux particuliers de conservation de la mémoire collective sont en danger, encore plus en ville, avec la croissance démographique et l'extension du tissu urbain souvent incontrôlé.

\section{Les mutations dans les comportements}

L’urbanisation a imprimé une certaine modernité au sein de la population de la commune de Koudougou, avec pour corollaire un changement dans les habitudes. Ainsi, les valeurs traditionnelles tendent à disparaître. La solidarité, par exemple, perd sa place au profit de l'individualisme. Autant de raisons qui confortent un chef de terre qui stigmatise l'urbanisation comme étant la base de la désorganisation sociale et de 
la dépravation des mœurs. Aussi, l'urbanisation suscite-elle une modernisation et un changement dans les comportements de la population. La gestion des bois sacrés s'en trouve modifiée. Un tel bouleversement de l'ordre établi par la société traditionnelle aura un impact sur l'évolution des coutumes et des traditions et sur le culte rendu dans les bois. En effet, il ressort des enquêtes que les générations actuelles s’intéressent de moins en moins aux cultes des ancêtres. Si bien qu'il est parfois difficile de trouver un héritier qui puisse poursuivre les rites. Le faible intérêt s'exprime déjà dans la conception que certains ont de ces reliques forestières: pour 44,8\% des personnes interviewées, elles ne présentent aucun intérêt, tandis que 34,3\% les perçoivent comme de simples espaces verts qui embellissent la ville.

\section{Les mutations dans les coutumes et traditions}

Les coutumes et traditions connaissent un changement dans leur pratique. Elles sont influencées par l'urbanisation, mais aussi par les religions monothéistes. En effet, bon nombre d'animistes se convertissent au christianisme ou à l'islam. Cela entraîne une dévalorisation de la religion traditionnelle. Du point de vue des responsables coutumiers, les cultes profitaient de la participation et de l'implication de tous les responsables de concessions. En plus de la participation au rituel du premier responsable, les membres sont également contraints au respect des prescriptions. Mais les transformations induites par les religions monothéistes ont réduit la participation des familles aux rituels dans les bois sacrés. Les rites coutumiers encore en vigueur sont devenus monotones, se raréfient et n'engagent plus que les garants de la coutume. La vie sociale organisée autour du culte traditionnel disparaît progressivement et prend d'autres formes; elle se définit autour de nouveaux axes d'expression des valeurs. Le bois sacré autour duquel la société se réunissait pour les fêtes de génération et les cérémonies d'initiation fait face à une crise de lisibilité.

L'urbanisation, mais davantage la modernité, assument toutes les deux une grande part de responsabilité dans les mutations qui affectent les coutumes et traditions. Sur huit chefs coutumiers interrogés, trois ont reçu une éducation formelle (école moderne) et deux appartiennent à d'autres confessions religieuses. Par conséquent, ils ont acquis une autre vision de l'environnement et de sa gestion. Ils sont souvent membres de l'administration moderne ou hommes politiques (enseignants, députés, etc.). Ainsi, ils manquent de temps pour jouer pleinement leur rôle de chefs traditionnels. Leurs conditions sociales (voitures, maisons luxueuses) les rapprochent davantage de la modernité, les éloignant donc de la tradition. Ce fossé qui se creuse entre les responsables coutumiers et les lois ancestrales contribue à perturber l'ordre social. Les mutations en cours ne sont cependant pas une forme d'adaptation de la coutume à l'urbanisation, mais plutôt une disparition progressive du traditionalisme en faveur du modernisme. Ce qui n'est pas sans effet sur la conservation des forêts sacrées. Cela ne préfigure-t-il pas une déterritorialisation de l'espace sacré, c'est-à-dire la dépossession des sites traditionnels sacrés au regard de la pression dont ils sont l'objet? Face au manque d'intérêt, le salut pourrait venir des structures modernes de gestion des territoires ou de l'environnement, mais ici également les caractéristiques des bois sacrés font que ces acteurs rechignent à en assurer le contrôle. 


\section{De la gouvernance moderne des bois sacrés aux nouvelles fonctionnalités}

\section{De nombreux acteurs avec des actions non synergiques}

La croissance démographique a progressivement réduit les caractéristiques rurales de Koudougou et de son hinterland, motivant ainsi de nouveaux mécanismes de gestion et de nouveaux acteurs agissant dans le cadre des collectivités territoriales. La gestion environnementale échoit, d'une part, à des services techniques de l'État ou des collectivités et, d'autre part, aux ONG (Yameogo, 2010). Les structures techniques de l'État en charge de la gestion des forêts et des espaces naturels sont constituées de la direction régionale, des directions provinciales, des services départementaux de l'environnement et du développement durable. Elles assurent la mise en œuvre des politiques et des orientations nationales à l'échelle locale. Il s'agit de la conservation, de la préservation et de la gestion harmonieuse des ressources forestières et faunistiques.

Àl'échelle communale, c'est une commission en charge des questions environnementales qui s'occupe de l'entretien des espaces verts de la ville et propose des stratégies d'aménagement de ces lieux. Il lui appartient aussi de veiller à ce que la ville ait un cadre de vie sain et de travailler à la restauration du couvert végétal. Cependant, cet engagement des structures étatiques et communales ne se limite qu'aux espaces non sacrés. D'autres acteurs, à l'image des organisations non gouvernementales (ONG) ${ }^{7}$ et des projets ${ }^{8}$, interviennent dans la conservation en prônant une approche participative qui inclut la population locale dans la gestion du patrimoine naturel. Cette approche se situe entre les logiques traditionnelles et modernes.

Malgré leur détermination à protéger l'environnement, aucune de ces structures ne s'est engagée à sauvegarder les forêts sacrées et leur biodiversité, bien que menacées de disparition. L'inaction à l'endroit de ces îlots de végétation vient de leur caractère sacré et des mythes dont ils sont auréolés. Une perspective de gestion participative des bois sacrés ne manque cependant pas de problèmes, comme le souligne Ibo (2005) dans l'analyse qu'il fait des activités de l'ONG intitulée «La croix verte dans les forêts sacrées en Côte d'Ivoire». En effet, les rituels sacrés dans les bois n’associent pas toujours les femmes. Pourtant, l'idée de la gestion participative nécessite que toutes les couches apportent leur contribution. Or, les lieux traditionnellement protégés, tels les bois sacrés, ne relèvent que de la compétence des coutumiers.

Les institutions modernes et les traditionnelles sont des organes indispensables au maintien de l'équilibre environnemental dans la commune de Koudougou. Quand bien même leurs logiques sont divergentes, l'aboutissement de leurs actions demeure le même. La gestion rationnelle des ressources naturelles et la conservation d'un minimum de verdure sont les objectifs communs, mais le manque de conjugaison des efforts entre les structures en charge de la protection de l'environnement et les responsables coutumiers est préjudiciable au maintien des bois sacrés.

7 Action Micro-Barrage (AMB), dans ce cas.

8 Par exemple, le Programme de développement local du Sanguié et du Boulkiemdé (PDL/SAB) et le Programme national de gestion des terroirs (PNGT). 


\section{Vers de nouvelles fonctionnalités des bois sacrés?}

Longtemps perçus comme des entités religieuses, les bois sacrés tendent à assumer de nouvelles fonctions dans le nouveau contexte urbain. De plus en plus, la population a une autre conception de ces espaces. Bien qu'ils doivent être préservés, les bois sacrés risquent, si ce n'est déjà le cas, de voir leur symbolique disparaître. Le magicoreligieux est la première et principale fonction que les forêts sacrées ont assumée depuis des temps immémoriaux. C'est d'ailleurs ce rôle qui leur a valu d'être conservées. Mais de nos jours, l'urbanisation a imprimé de nombreuses mutations dans la société et une dynamique au sein de ces bois qui tendent à les désacraliser. De ce fait, de nouveaux rôles leur sont alors conférés et tendent à montrer que le rapport au sacré peut induire de nouvelles stratégies d'organisation et de gestion à travers des fonctionnalités innovantes d'embellissement, sanitaire, pédagogique et patrimoniale.

\section{La fonction d'embellissement}

Les forêts sacrées sont considérées comme des espaces d'ornement pour la ville de Koudougou. Ce nouveau rôle leur est attribué par 24,1\% des personnes interrogées. En effet, compte tenu de la faible densité de végétation dans la ville, la concentration d'arbres et d'arbustes en un lieu constitue un cadre verdoyant agréable. Le service provincial de l'environnement et du développement durable accorde à cette fonction un intérêt particulier. Pour cette structure, un centre urbain a besoin de ce type d'enclaves boisées pour l'embellissement et l'ornement du cadre de vie. Il préconise de ce fait une urbanisation modérée avec conservation d'espaces verts. En outre, la municipalité a tout intérêt à assurer la préservation de ces îlots, d'autant plus que les espaces verts prévus dans les plans de lotissement de la ville sont rarement verts. Sur près de 50 espaces verts dénombrés pendant les lotissements, très peu abritent encore de la végétation.

\section{La fonction sanitaire}

Les reliques forestières sacrées jouent un rôle très important dans la santé de la population, selon $27,5 \%$ des participants à l'enquête. Elles contribuent à réduire les infections pulmonaires à travers leur pouvoir d'absorption des gaz nuisibles $\left(\mathrm{CO}^{2}\right)$. Les effets induits des nombreuses pollutions urbaines (rejet de gaz dans l'atmosphère par les usines, les trains, les voitures, les motos et autres engins à moteur) et des poussières sur la santé sont limités grâce à la présence de végétation. De ce fait, les forêts sacrées seraient des appareils d'épuration de l'air au sein de la ville.

\section{La fonction pédagogique}

Ces enclaves de biodiversité dans la zone d'étude sont bénéfiques à plus d’un titre. Elles contribuent à l'édification de la connaissance écologique, en offrant la possibilité aux apprenants (élèves, étudiants) et chercheurs de découvrir sur place certaines espèces et d’y mener des recherches. Les nouvelles fonctions assumées par les forêts sacrées sont des raisons qui justifient la nécessité de leur existence en ville. L'intérêt n'est plus seulement d'ordre coutumier et socioculturel, mais ces espaces sont devenus, de par leurs nouveaux rôles, des lieux d'épanouissement. Cet aspect devrait motiver davantage leur sauvegarde. 


\section{La fonction patrimoniale}

Selon la définition du conseil de l'Europe ${ }^{9}$, le patrimoine est «un ensemble de ressources héritées du passé que des personnes considèrent, par-delà le régime de propriété des biens, comme un reflet et une expression de leurs valeurs, croyances, savoirs et traditions en continuelle évolution. Cela inclut tous les aspects de l'environnement résultant de l'interaction dans le temps entre les personnes et les lieux». Ainsi, un objet patrimonial traduit des valeurs conservées à transmettre aux générations futures. En cela, les bois sacrés méritent donc le qualificatif de patrimoine. En effet, les entretiens avec les acteurs en charge de la gestion des ressources naturelles font ressortir le fait que chacun reconnaît la nécessité de la conservation et de la protection des bois sacrés. Ce capital social ${ }^{10}$ autour des bois sacrés peut alors être mis à profit pour aller vers une gestion patrimoniale, dans l'objectif de satisfaire l'ensemble des acteurs bénéficiaires d'intérêts partagés (Neira, 2004). Pour Revéret et Webster (2002), «l'approche patrimoniale est un état d'esprit permettant au plus grand nombre de titulaires d'un patrimoine de garder le souci du long terme, de prendre conscience des multiples interdépendances entre acteurs, et de se rencontrer pour décider en commun des mesures qui permettront, au plus juste prix, de conserver ou d'augmenter ce patrimoine tout en tirant la plus grande quantité possible d'avantages».

Les défenseurs des bois sacrés, soucieux de la préservation de leurs sanctuaires, approuvent cette approche patrimoniale des ressources que leurs ancêtres se sont évertués à protéger, qu'ils leur ont transmise et qu'eux aussi entendent transmettre aux générations futures. De même, leur maintien constitue une empreinte importante pour l'identité culturelle des lignages fondateurs, détenteurs des rites sacrificiels dans le bois. L'inscription des bois sacrés au statut de "patrimoine urbain» leur permet d'échapper aux différentes formes de pression dont ils sont l'objet. Mais le nouveau contrat social avec l'ensemble des acteurs favorise davantage le maintien des mythes, des symboles et des valeurs ancestrales. En marge de cette reconnaissance de la fonction religieuse par la patrimonialisation, c'est également la perspective fonctionnelle des bois sacrés, défendus par les services en charge de l'environnement de l'État et de la commune de Koudougou ainsi que par des ONG qui se trouve valorisée.

\section{Les conditions d'une réhabilitation et d'une gestion concertée des bois sacrés}

Les modes de gestion traditionnels qui ont permis le maintien des forêts sacrées dans leur état actuel sont aujourd'hui bouleversés. Les coutumiers, garants de ces espaces, ont perdu en partie l'autorité qui leur aurait permis de faire respecter les interdits et de préserver les bois sacrés. À l'inverse, les structures modernes possèdent l'autorité nécessaire à la gestion, mais ont du mal à s'y intéresser au regard des mythes qui entourent encore ces espaces. Toutefois, chacun des acteurs reconnaît le rôle de ces îlots et la nécessité de les préserver. Quelles sont alors les possibilités d'aménagement et les stratégies à mettre en œuvre?

9 La Convention de Faro sur la valeur du patrimoine culturel pour la société, 27 octobre 2005.

10 Le capital social représente l'ensemble des règles et des capacités d'organisation qui coordonne le comportement humain, notamment dans l'usage des ressources (Hanna et Jentoft, 1996). 


\section{Les possibilités coutumières}

Conscients des menaces qui pèsent sur leurs sanctuaires, les coutumiers n'excluent pas l'idée d'une cogestion impliquant les autorités communales. Cette position traduit déjà une certaine flexibilité, une capacité d'adaptation face aux transformations de la ville et une évolution de la relation au sacré qui pourrait induire des aménagements, de nouvelles plantations, par exemple. Cependant, il faut se garder de tout optimisme béat du fait que le respect du sacré reste ancré dans l'imaginaire collectif, et ce, en dépit de l'adoption des religions monothéistes. Même si les citoyens ne pratiquent plus le culte des ancêtres, ils n'iront pas jusqu'à coloniser ces espaces. En outre, en dépit des empiètements sur leurs marges, les citadins rechignent encore à investir totalement les bois sacrés. Ce qui fait dire que le sacré semble difficile à délocaliser. En effet, même lorsque certains sites ne sont plus investis par la végétation, la sacralité de l'espace demeure; au point que personne ne veut y habiter ou les aménager. Aussi, cela constitue-t-il des perspectives d'aménagement.

\section{Les possibilités au sein des administrations}

Du point de vue des administrations chargées de la gestion de l'environnement urbain, la destruction des espaces sacrés n’est pas envisagée. Il n’en demeure pas moins que leur symbolique mérite d'être revue afin d'en permettre l'aménagement. Les interventions dans ce cadre pourront s'articuler autour de l'érection de clôtures, de reboisements complémentaires, etc., afin de les soustraire à la dégradation. On le voit bien, les différents acteurs ne s'excluent pas, mais c'est plutôt le cadre de rencontre pour débattre ces questions qui manque. Cependant, des actions semblent avoir été prises par le service municipal en vue d'un meilleur entretien des bois sacrés. Il s'agit notamment d'activités de sensibilisation, de salubrité et de reboisement. Ces actions peuvent être considérées comme des prémices d'aménagement. La population locale est également favorable à une conservation des reliques forestières, même par des modifications. Ainsi, 72,4\% des personnes interrogées préconisent des clôtures tandis que, pour $27,6 \%$ des interlocuteurs, il faut en plus impliquer tous les acteurs de la protection de l'environnement dans la gestion de ces espaces. Le dernier défi est financier et semble le plus difficile à relever.

\section{Les perspectives d'une gestion concertée}

La préservation des ressources naturelles, notamment les îlots de végétation sacrée en milieu urbain, demeure une composante essentielle de la biodiversité. Mais à l'épreuve du bâti et du béton, la symbolique des bois sacrés, et plus généralement du sacré, mérite d'être repensée par les acteurs intéressés, pour lui donner une nouvelle vocation. Cela ne passe-t-il pas par un renforcement des capacités des acteurs et par une allocation de ressources financières pour l'aménagement des bois sacrés? Au-delà des ressources financières à injecter, les autorités coutumières ont besoin d'être sensibilisées et associées aux initiatives d'aménagement et de valorisation (Cormier-Salem et al., 2005). On assisterait à une reformulation du sacré vers un «sacré laïc» reconnu par la société entière (Raffestin, 1985: 104) comme un patrimoine à préserver. L’aménagement des bois sacrés pourrait permettre de les transformer en lieux touristiques. Les exemples de sites sacrés devenus des sites d'attraction 
foisonnent à souhait au Burkina, pour ne citer que les mares aux caïmans sacrés de Sabou, aux silures sacrés de Dafra et aux hippopotames sacrés de Tengrela. Le parc urbain Bangr-Weoogo de Ouagadougou, jadis forêt sacrée, n’a pas perdu toute sacralité, en dépit des aménagements et des activités sportives et récréatives qui s’y déroulent. Il bénéficie d'une gestion conjointe entre la mairie, le service forestier et les coutumiers (Reuse, 1999). À Koudougou, cette perspective est envisageable, car les différents acteurs n'y voient aucun inconvénient. Ainsi, d’objets spirituels vénérés, les bois sacrés deviennent des objets fonctionnels et tombent progressivement dans le patrimoine urbain des communes.

\section{Conclusion}

L'harmonie des relations entre l'homme et le milieu naturel est fondamentale pour garantir la pérennisation des ressources de l'environnement. Mais cette harmonie est perturbée par l'urbanisation, qui engendre des manquements aux règles préétablies par la société traditionnelle pour la conservation des bois sacrés dans la commune de Koudougou. Ces violations causent de graves dommages qui hypothèquent l'avenir des bois sacrés. Cependant, l'adoption de certaines mesures permettrait de parer à la disparition de tels massifs. Dans cette perspective, il serait nécessaire que les pouvoirs publics apportent le soutien indispensable aux mesures de protection de la nature. Par ailleurs, la sauvegarde durable de l'environnement requiert l'engagement volontariste et la participation franche de la population. L'engagement affiché des gardiens des bois sacrés à accompagner les autorités municipales dans une gestion concertée présage ainsi d'une mutation du sacré vers le fonctionnel.

\section{Remerciements}

L’auteur remercie Bayala Eric pour la cartographie et les photos. 


\section{Bibliographie}

AGNISSAN, Aubin (2010) Dynamique urbaine et conservation des forêts sacrées: enjeux théoriques et paradoxes épistémologiques. Communication présentée au Colloque international BiodiverCities.

BARRETEAU, Daniel, DOGNIN, René et VON GRAFFENRIED, Charlotte (1991) L'homme et le milieu végétal dans le bassin du Tchad. Éditions ORSTOM.

BOUKPESSI, Tcha (2010) Les pratiques endogènes de conservation de la biodiversité au Centre-Togo. Université de Lomé et de Franche-Comté, thèse de doctorat unique de géographie.

CIRAD (CENTRE DE COOPÉRATION INTERNATIONALE EN RECHERCHE A GRONOMIQUE POUR LE DÉVELOPPEMENT) (2001) Les forêts tropicales dans la vie des hommes. Rapport.

CORMIER-SALEM, Marie-Christine, JUHÉBEAULATON, Dominique, BOUTRAIS, Jean et ROUSSEL, Bernard (2005) Patrimoines naturels au sud, identités et stratégies locales. Paris, IRD Éditions.

DEFFONTAINES, Pierre (1948) Géographie et religions. Paris, Gallimard.

DUCHESNE, Véronique (2002) Des lieux sacrés aux sites classés. Évolution du contrôle des ressources naturelles dans le Sud-Est ivoirien. Dans Marie-Christine Cormier-Salem, Dominique Juhé-Beaulaton, Jean Boutrais et Bernard Roussel (dir.) Patrimonialiser la nature tropicale. Dynamiques locales, enjeux internationaux. Paris, IRD Éditions, p. 419-438.

GANABA, Souleymane, OUADBA, Jean Marie et BOGNOUNOU, Ouetian (1998) Les ligneux à usage de bois d'énergie en région sahélienne du Burkina Faso: préférence des différents groupes ethniques. Sécheresse, vol.9, nº4, p. 261-268.
HANNA, Susan et JENTOFT, Svein (1996) Human use of the natural environment: An overview of social and economic dimensions. Dans Susan Hanna, Carl Folke et Karl-Göran Mäler (dir.) Rights to nature, ecological, economic, cultural and political principles of the institutions for the environment. Washington D.C., Island Press, p. 35-55.

HIEN, Héliane M. (2007) Rôle social et culturel de l'artisanat face à la déforestation: cas des artisans de Ouolonkoto (province $d u$ Houet). Université de Ouagadougou, mémoire de maîtrise de sociologie.

IBO, Jonas (2005) Contribution des organisations non gouvernementales écologistes à l'aménagement des forêts sacrées en Côte d'Ivoire: l'expérience de la Croix Verte. VertigO - La revue en sciences de l'environnement, vol. 6, $\mathrm{n}^{\circ} 1$ [En ligne]. http://vertigo.revues.org/2813

JUHÉ-BEAUlATON, Dominique (2006) Enjeux économiques et sociaux autour des bois sacrés et la "conservation de la biodiversité». Bénin, Burkina Faso, Togo.

JUHÉ-BEAULATON, Dominique (2009) Un patrimoine urbain méconnu: arbres mémoires, forêts sacrées et jardins des plantes de Porto Novo (Bénin). Autrepart, n 51.

KABORÉ, Oger (1987) L'arbre dans la pensée symbolique chez les Moose: l'exemple du néré, du karité et de l'acacia albida. Communication au Séminaire national sur les essences forestières locales, 6-10 juillet, Ouagadougou, Burkina Faso, p. 34-45.

KABORÉ, T. (1994) "Crise» de l'arbre et gestion paysanne des ressources arborées: l'exemple de la zone d'encadrement agricole de Zaamsé. Université de Ouagadougou, mémoire de maîtrise de sociologie.

KONSEGRÉ, Hortense (2007) Les transports urbains de Koudougou. Université de Ouagadougou, mémoire de maîtrise de géographie. 
MECV (MINISTÈRE DE L'ENVIRONNEMENT ET DU CADRE DE VIE) (2006) Plan d'action de mise en œuvre des réformes institutionnelles et juridiques pour la décentralisation dans le secteur forestier.

NEIRA, Francisco Brito (2004) Appropriation des ressources naturelles dans une forêt andine équatorienne: vers la gestion patrimoniale des ressources. VertigO La revue en sciences de l'environnement, vol. 5, no 2 [En ligne]. https://vertigo. revues.org/3573

ONU-HABITAT (2005) Profil urbain national du Burkina Faso.

PÉLISSIER, Paul (1980) L'arbre en Afrique tropicale: la fonction et le signe. Préface Cahiers ORSTOM. Série Sciences humaines, vol. 17, nos 3-4, p. 127-130.

RACINE, Jean-Bernard (1993) La ville entre Dieu et les hommes. Paris, Anthropos, Genève, Presses bibliques universitaires.

RACINE, Jean-Bernard et WALTHER, Olivier (2003) Géographie et religions: une approche territoriale du religieux et du sacré. SEDES/VUEF - L'Information géographique, vol. 67, n³, p. 193-221.

RAFFESTIN, Claude (1985) Religions, relations de pouvoir et géographie politique. Cahiers de géographie du Québec, vol.29, no 76, p. 101-107 [En ligne]. http:// id.erudit.org/iderudit/021697ar

REUSE, Stéphane (1999) Étude d'un projet urbain dans le contexte de la ville africaine: le cas de "Ouaga 2000». Université de Lausanne, Institut de géographie.

REVÉRET, Jean-Pierre et WEBSTER, Alain (2002) Economics and biodiversity management. Dans Philippe Le Prestre (dir.) The construction of a new international biological order. Aldershot, Hampshire Ashgate Publishing Ltd.

TURCO, Angelo (1988) Verso una teoria geografica della complessità. Milano, Unicopli.
YAMEOGO, Lassane (2010) Diversité d'appropriation des approches participatives dans la gestion des aires protégées de Gonsé et de la Comoé-Léraba (Burkina Faso). Cahiers du CERLESHS, Tome XXV, nº35, p. 45-69. 